JOURNAL DE PHYSIQUE

Colloque C10, supplément au $\mathrm{n}^{\circ} 12$, Tome 46 , décembre 1985 page $\mathrm{Cl0}-179$

\title{
DISLOCATION-SOLUTE ATOM INTERACTION IN ALUMINUM STUDIED BY AMPLITUDE-DEPENDENT INTERNAL FRICTION
}

\author{
T. KOSUGI AND T.KINO \\ Laboratory of Crystal Physics, Faculty of Science, Hiroshima \\ University, Hiroshima, Japan
}

\begin{abstract}
In order to get the information on the force-distance curve for the interaction between a dislocation and a solute atom, amplitude-dependent internal friction was measured at 1.7 - $30 \mathrm{~K}$ on $\mathrm{A} 1-100$ at ppm $\mathrm{Zn}$ and $\mathrm{A} 1-50$ at ppm Zn single crystals. The temperature dependence of applied strain under a constant decrement is the same for two concentrations with high accuracy. The data were analyzed by the thermally assisted break-away"model for dislocations assuming several potential forms. In conclusion, a modified Cottrell expression is proposed, in which dislocation core structure is taken into account, and the model can explain the temperature dependence of applied strain satisfactorily. The core radius of dislocation is estimated to be about $3 \AA$.
\end{abstract}

\section{I - INTRODUCTION}

Dislocations in crystals interact with various kind of particles, for example, point defects, electrons, phonons and so on, because of the structural disorder caused by them. The details of the interactions strongly reflect the structure of a dislacation.

The ultrasonic method proposed by Schwarz and Granato/l/ is a very useful one to get the information on the details of the interaction between a dislocation and a point defect. We used this method, i.e., the measurement of amplitude-dependent internal friction due to dislocation break-away from pinning points at low temperatures, to get the force-distance curve for the interaction between a dislocation and a single solute atom.

II - EXPERTMENTAL

Specimens are $A 1-100$ at ppm $\mathrm{Zn}$ and $\mathrm{Al}-50$ at ppm $\mathrm{Zn}$ single crystals $\left(3.5 \times 3.5 \times 50 \mathrm{~mm}^{3}\right.$ with [311] and [412] axes, respectively) made by a strain-annealing method, and annealed at $500^{\circ} \mathrm{C}$ for $3 \mathrm{~h}$ in air and slowly cooled in a furnace to room temperature before the measurement. We selected these specimens from following reasons: the desired information will be obtained by introducing a single kind of solute atom with very low concentrations, especially zinc in $\mathrm{Al}$ is entirely dispersed on the dislocation lines as a single atom and further inertial effect/2/ or line tension effect/3/ may not occur because of low pinning concentrations. Amplitude-dependent internal friction measurements were carried out by a composite oscillator method (51 kHz) at $1.7-$ 
$30 \mathrm{~K}$. The accuracy of the measurement was better than $1 \%$. Details of the experimental procedure are similar to the previous work $/ 4 /$.

Internal friction caused by dislocation break-away from pinning points is stress (or strain) amplitude dependent loss. Firstly we confirmed this point as shown in Fig. 1. The strain level at which the amplitude dependence started is larger for Al100 at ppm $\mathrm{Zn}$ than for A1-50 at ppm Zn, by a factor about 1.5. Here the background loss (amplitude independent loss) is explained as the interaction of acoustic wave with conduction electrons from the difference of the loss between two specimens. Thus we next measured the temperature dependence of applied strain under a constant internal friction in a region where the amplitude dependent loss is dominant (Fig. 2). The influence on the value of $\varepsilon$ from the change of background loss with. temperature was negligible, i.e., at most less than $2 \%$ near $30 \mathrm{~K}$ for $\mathrm{Al}-50$ at $\mathrm{ppm} \mathrm{Zn}$. The accu-

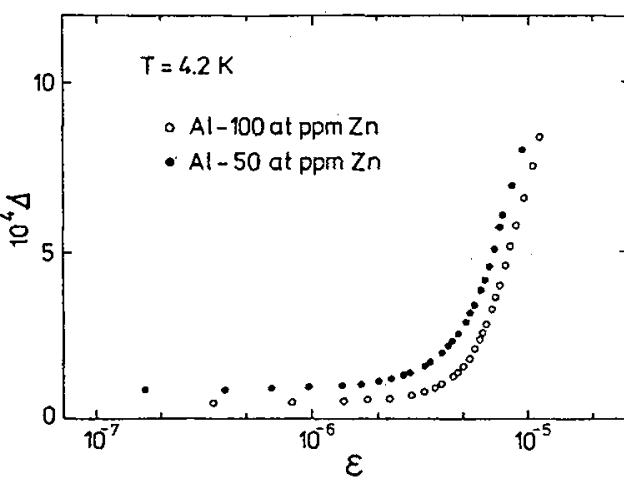

Fig. 1 Applied strain amplitude dependence of internal friction at $4.2 \mathrm{~K}$. racy and reproducibility of the measurement is very high for each specimen, and furthermore the temperature dependence of $\varepsilon$ is the same for two specimens with different concentrations. This indicates the obtained curves precisely reflect the force-distance curve for the interaction between a dislocation and a single $\mathrm{Zn}$ solute atom.

\section{III - DISCUSSION}

In the case that dislocations are pinned by point defects along the dislocation segments and the break-away starts from the pinning point at the center of maximum double loop in each segment, the stress (or strain) amplitude required to produce a constant decrement was shown by Schwarz and Granato to follow the $\mathrm{T}^{2} /{ }^{3}$ law assuming the parabolic

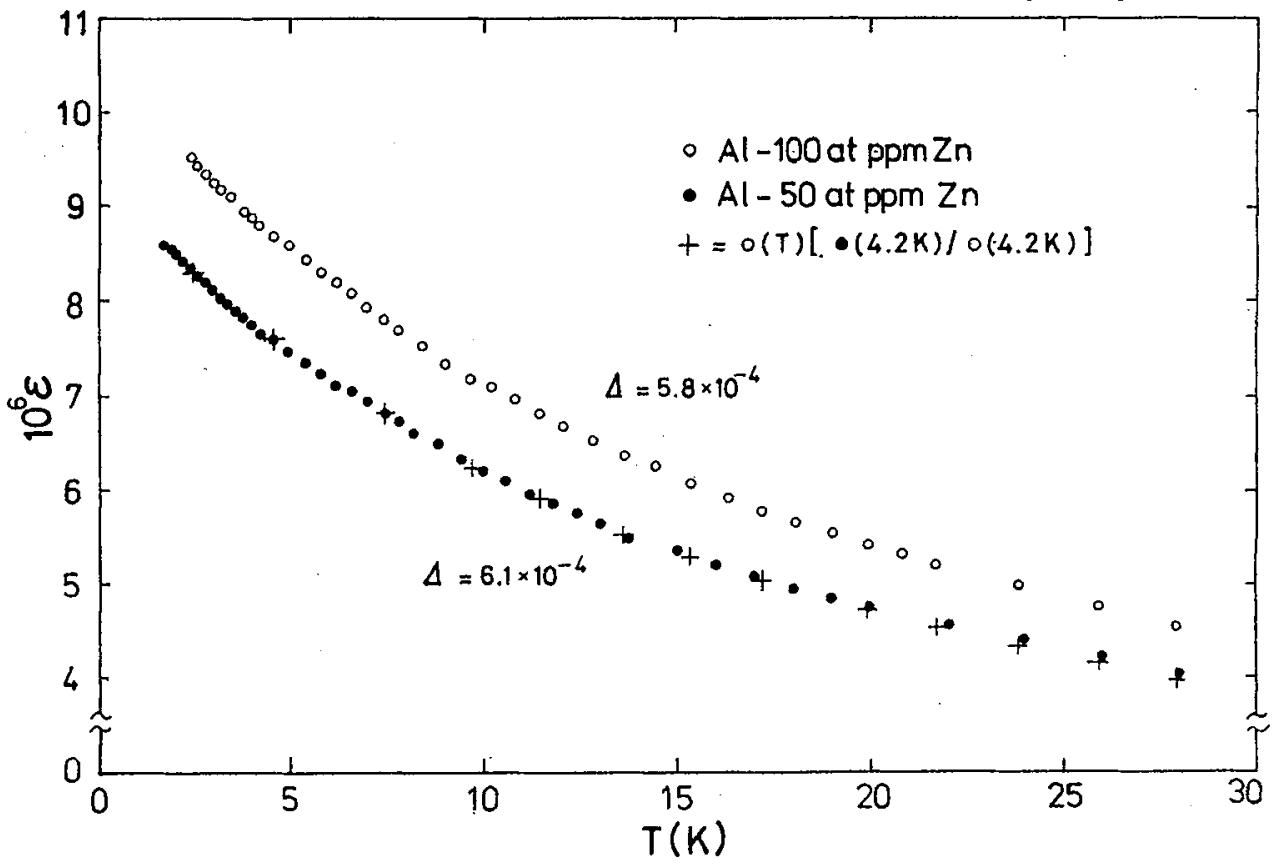

Fig. 2 Temperature dependence of applied strain under a constant internal friction. The symbol + shows the values of $\varepsilon$ for $A 1-100$ at $\mathrm{ppm} \mathrm{Zn}$ divided by the ratio of $\varepsilon$ for A1-100 at ppm $\mathrm{Zn}$ to $\mathrm{A} 1-50$ at ppm $\mathrm{Zn}$ at $4.2 \mathrm{~K}$. 


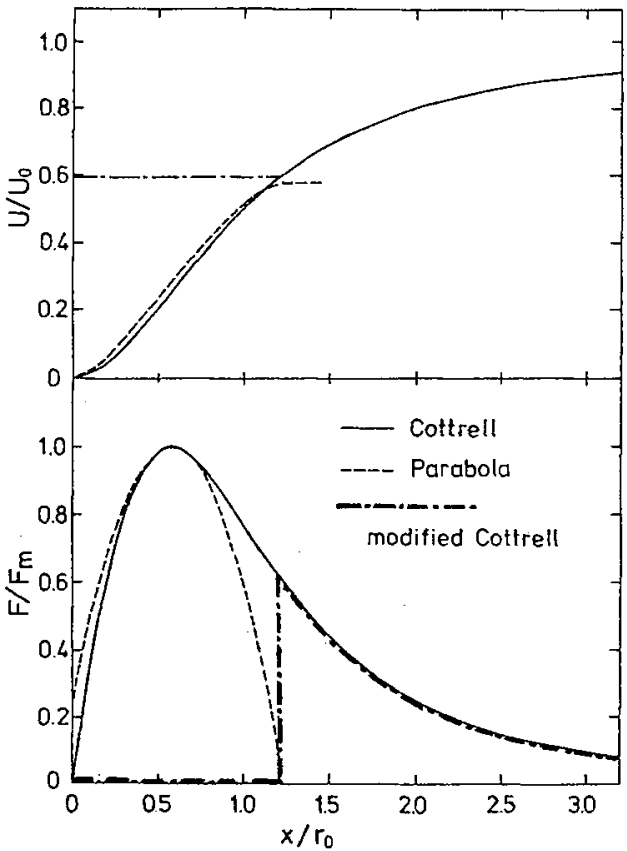

Fig. 3 Interaction energy-distance curves and force-distance curves. Here $r_{0}$ is the distance of closest approach of solute atom to dislocation center. force-distacnce curve/1/. According to the precise treatment by Granato and Lücke, the coefficient of $\mathrm{T}^{2 / 3}$ is not strictly constant but depends on $T / 5 /$. We analyzed our data using the formula ( $A-9$ ) in their paper $/ 5 /$ assuming the Cottre 11 expression/6/ and its parabolic approximation for the force-distance curve (Fig. 3). We note here that the force-distance curve for the Cottrell expression is very reasonable to assume, since the apparent form is the same as those for the modulus effect (the other elastic interaction) and the electrical interaction when the dislocation moves on the glide plane. Fitting parameters are only two, i.e., $\varepsilon_{M}$ ( $\varepsilon$ at $\mathrm{T}=0$ ) and the binding energy, $\mathrm{U}_{0}$ and $\mathrm{U}_{1}\left(\mathrm{U}_{1}=0.577 \mathrm{U}_{0}\right)$ for the Cottrell expression and its parabolic approximation, respective1y. The parabolic form is a good approximation near the maximum force $F_{m}$ of the Cottrel1 expression, i.e., at a low temperature or $F / F_{m} \geq 0.85$. Thus we tried to fit the calculated curves with experimental data satisfying $\varepsilon / \varepsilon_{M}$ (or $\left.F / F_{m}\right) \geqq 0.85$. Calculations were carried out with corrections for the distribution of maximum double loop length in each dislocation segment and the inhomogeneity of strain amplitude along the specimen rod. The fitting with the parabolic approximation (best fitting value $\mathrm{U}_{1}=0.035$ $\mathrm{eV})$ is not good for $\mathrm{T} \geqq 5 \mathrm{~K}\left(\varepsilon / \varepsilon_{\mathrm{M}} \leqq 0.8\right)$,

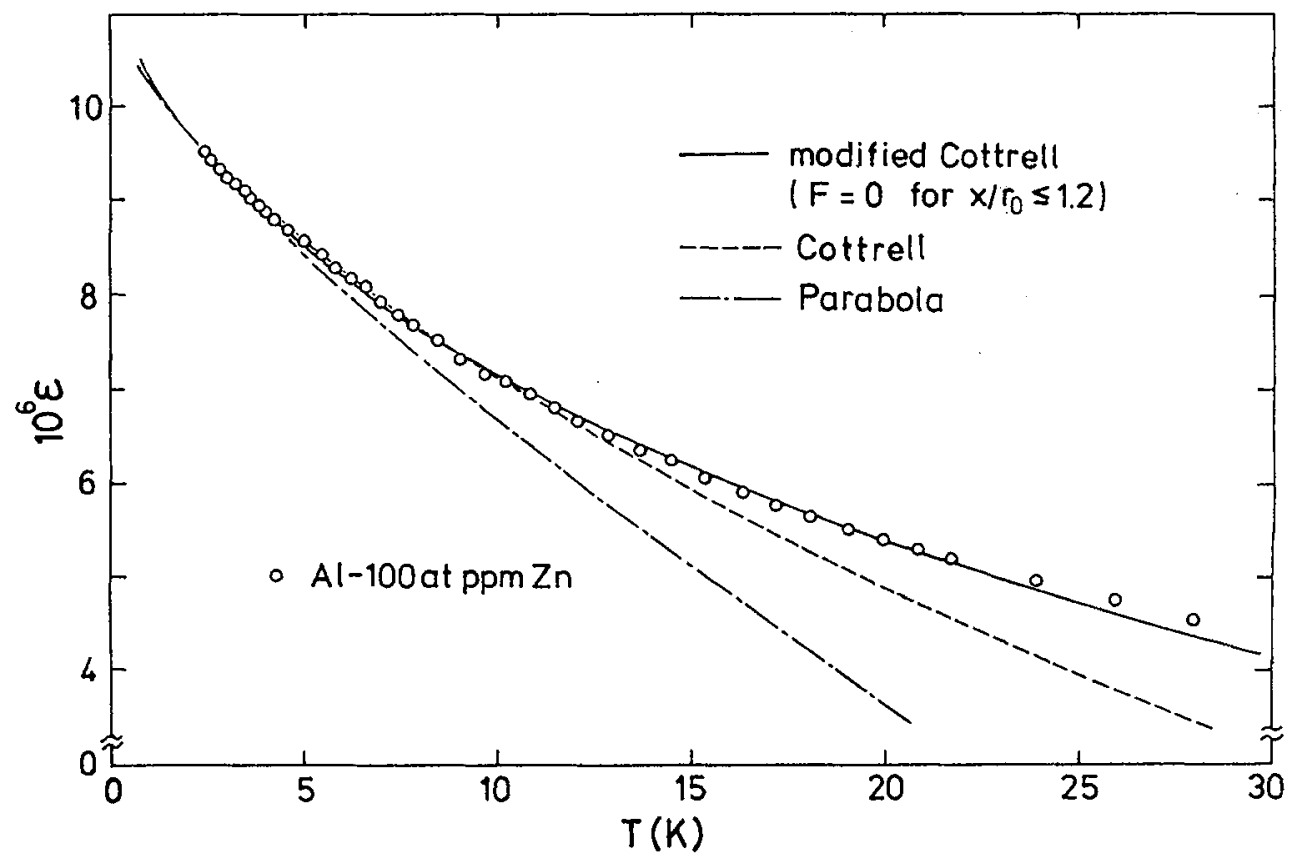

Fig. 4 Temperature dependence of applied strain. Calculations from the Cottrell expression, its parabolic approximation and the modifier. Cottrell expression are compared with the experiment. 
while the Cottrell expression $\left(\mathrm{U}_{0}=0.061 \mathrm{eV}\right)$ gives a good fitting until further higher temperature, i.e., $13 \mathrm{~K}\left(\varepsilon / \varepsilon_{\mathrm{M}} \simeq 0.6\right)$ as seen in Fig. 4. However, above $13 \mathrm{~K}$, experimental values gradually deviate from the calculated curve for the Cottrell expression. This means the real force-distance curve differs from the Cottrell type one.

In order to overcome this problem, we considered two mechanisms, one is the effect of line tension (LTE) and the other is the effect of dislocation core structure (DCE). As well known, a dislocation has the line tension. If a dislocation loop bows gut by a distance $\mathrm{x}$ with the applied stress $\sigma$, the line tension energy increases by $\mathrm{Cx}^{2} / 1$ (C: line tension, 1: loop length). Therefore, the broken-away state becomes unstable for a short double loop of dislocation at a larger $\sigma / \sigma_{M}$ (or $\varepsilon / \varepsilon_{M}$ ) than for a long one because of the line tension energy/3/. If the stress is smaller than a critical value $\sigma^{*}$, break-away can no longer take place for a double loop as stated by Granato and Lücke/5/. In such a case, break-away must occur simultaneously at two or more pinning points, and thus the higher activation energy is necessary and the decrease of $\varepsilon$ with increasing temperature becomes slower than the double-loop depinning. However, in our measurement, no difference in the temperature dependence between Al-100 at ppm $\mathrm{Zn}$ and Al-50 at ppm $\mathrm{Zn}$ was observed, therefore the dislocation loop length for the double-loop depinning is probably so large that LTE is negligible. Next we consider DCE. Near the dislocation center, elastic continuum theory is not available as a usual saying. However, the DCE has not been observed in any experiments until now, at least in fcc metals. There seems to be only computer simulations as precise investigations, for instance, that by Cotterill and Doyama for edge dislocation core structure in $\mathrm{Cu} / 7 \%$. According to their results, in the dislocation core region elastic continuum theory is no more available and relaxation occurs to lower the core strain energy. For simplicity, we considered a modified Cottrell model, in which elastic continuum theory holds for the distance $\mathrm{x}$ larger than a critical distance $x_{c}$, within which the interaction energy $U$ or the stress field of dislocation is constant for discreteness of lattice as shown in Fig. 3. It was found that the modified Cottrell expression can explain the obtained temperature dependence of $\varepsilon$ satisfactorily, if we select an appropriate $x_{c}\left(1.2-2.0 r_{0}\right)$ as seen Fig. 4. Fitting goodness is gradually poorer when $x_{c}$ is taken smaller value than $1.2 \mathrm{r}_{0}$. On the other hand, when $\mathrm{x}_{c} \geqq 1.2 \mathrm{r}_{0}$, the goodness does not seem to change so much. However we cannot take the value of $x_{c}$ as much larger than $x_{c}=1.2$ ro, because if so, we overestimate the elastic interaction or the apparent $U_{0}$ (see Fig. 3) to fit the calculated curve with experiments.

In the case we take $x_{c}=1.2 r_{0}$, determined parameter values are as follows. The binding energy for the modified Cottrell expression $U_{0}^{3 / 2}=0,11 \mathrm{eV}$ (the apparent $\left.\mathrm{U}_{0}=0.27 \mathrm{eV}\right)$, and $\mathrm{x}_{\mathrm{c}}=1.4 \AA\left(\mathrm{r}_{0}=1.17 \AA\right)$. From $\mathrm{U}_{0}=0.27 \mathrm{eV}$, the misfit parameter $\delta\left(=\left(a_{\mathrm{Al}}-\mathrm{a}_{\mathrm{Zn}}\right) / \mathrm{a}_{\mathrm{A1}}\right.$; a: atomic radius $)$ is estimated as 0.06 which is reasonable. Furthermore, the estimation for the core radius $r_{c}$ within which elastic continuum theory gannot hold, was made assuming $1 / r_{c}=r_{0} /\left(r_{0}^{2}\left\{+x_{c}^{2}\right)\right.$, and resulted in $r_{0}$ being about $3 \mathrm{~A}$. We note, in fcc metals, there has been no other experiment observing the dislocation core effect and getting the information on the core structure.

Our study was made to get the information on a dislocation-a solute atom interaction, and furthermore obtained results show the knowledge on the dislocation core structure stated above.

Acknowlegement - We wish to express our gratitude to $T$. Nakamura for his help in the experiments and calculations.

\section{REFERENCES}

/1/ Schwarz, R, B, and Granato, A. V., Phys. Rev. Lett. 34 (1975) 1174.

/2/ Isaac, R. D., Schwarz, IR. B. and Granato, A. V., Phys. Rev. B18 (1978) 4143.

/3/ Teutonico, L. J., Granato, A. V. and Lücke, K., J. App1. Phys. 35 (1964) 220.

/4/ Kosugi, T. and Kino,. T., J. Phys. Soc. Jpn. 53 (1984) 3837.

/5/ Granato, A. V. and Lücke, K., J. App1. Phys. 52 (1981) 7136.

16/ Cottrell, A. H., Dislocations and Plastic Flow in Crystals (Oxford Univ. Press, London, 1953) p 139.

/7/ Cotterill, R. M. J. and Doyama, M., Phys. Rev, 145 (1966) 465. 